\title{
Dielectric Method for Diagnosing Formation of Gas Hydrates in Gas Pipelines
}

\author{
Sergey Podorozhnikov" ${ }^{1, *}$, Alexander Shabarov, ${ }^{2}$ Albina Shirshova ${ }^{2}$, and Artyom \\ Berdyshev $^{2}$ \\ ${ }^{1}$ Industrial University of Tyumen, 625001, Volodarskogo str. 38, Tyumen, Russia \\ ${ }^{2}$ Tyumen State University, 625001 Volodarskogo st. 6, Tyumen, Russia
}

\begin{abstract}
Experimental study of low-frequency dependence of the dielectric properties of gas hydrate in the process of growth and dissociation in the range $50-1000 \mathrm{kHz}$ is conducted. It is found that the dielectric loss tangent maximum value is observed at a frequency of 260 $\mathrm{kHz}$ and depends on the amount of gas hydrate in the measuring cell. Comparison of degrees of gas-hydrate formations obtained by two independent methods (PVT-method and dielectric method) showed good agreement. It allows us to offer a simple method of controlling the process gas-hydrate formations in the pipes based the measurement of the dielectric loss tangent at a certain frequency.
\end{abstract}

\section{Introduction}

It is known that natural gas in certain thermodynamic conditions enter into combination with water to form hydrates, which are accumulating in the field and main pipelines, significantly increase its hydraulic resistance and hence reduce capacity. The problem of combating the hydrates formation becomes particularly important while developing deposits in Western Siberia and the Far North. Low temperatures and severe climate conditions in these areas provide favorable conditions for hydrate formation in wells and pipelines (see. Figure 1).

For the destruction of hydrates accumulations, the method of applying electromagnetic waves of microwave range is very efficient [2]. For example, in [3] the results of field tests of this method at a special test section (length $16 \mathrm{~m}$ ) flowline connected to one of the wells Efremovsky deposits of Kharkov GPU are shown.

\section{Object of research (model, process, configuration, synthesis, experimental part, etc.)}

Diversity of wells and pipes equipped for production and transportation of oil and gas requires the development of a number of fundamentally different methods and devices for

\footnotetext{
* Corresponding author: sergey urevih@mail.ru
} 
HF EMF actuation in pipes and wells, in which the different obstructions are formed. Depending on the design and parameters of wells and pipelines, it is necessary to choose a specific technological scheme of electromagnetic exposure and the necessary range of the electromagnetic equipment. However, their physical basis is made by transformation of electromagnetic energy part either in intertube space, or inside the tubes into heat due to the finite conductivity of pipes materials and dielectric losses of the area of filling intertube space and pipes.

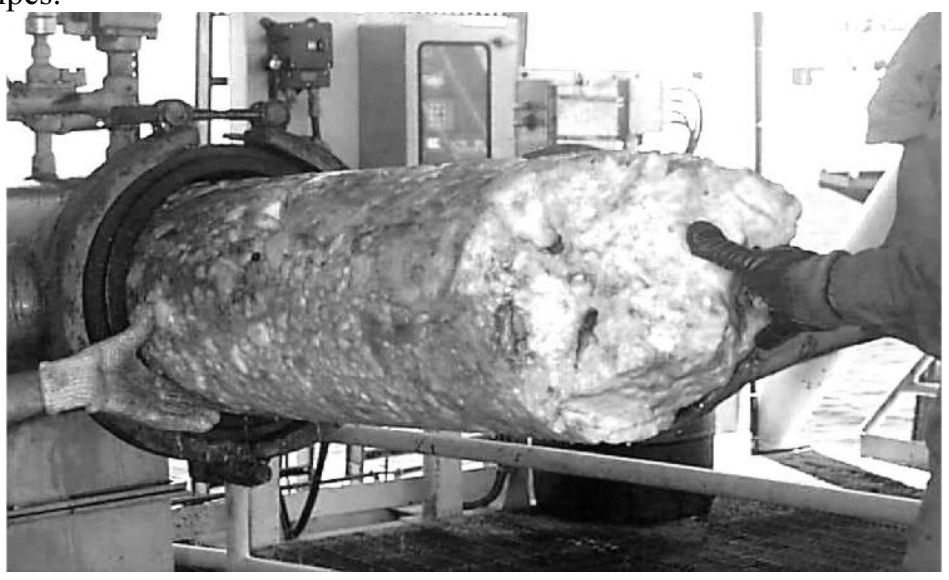

Fig. 1. Hydrate plug in the pipeline [1].

Researches [4], [5], [6], [7], [8], [9] are devoted to theoretical and experimental investigation of the dielectric properties of gas hydrates. However, these works mainly represent the results of researches in high-frequency electromagnetic field, where energy is converted to heat, sufficient for decomposition of gas hydrate.

On the other hand, the electronic response to the impact of low frequency electromagnetic field of low power and gas hydrate can provide useful information about the beginning of the process of gas-hydrate formation in pipe, thus avoiding accidents and pipeline downtime. Furthermore, this information can quantify the degree of gas-hydrate formation, not destroying the formed gas hydrate.

\section{Methods}

The objective of this paper is to provide a method of control the gas hydrate formation process using dielectric method.

Tasks:

1. Experimental study of hydrate dielectric parameters in the process of its formation and dissociation in the low-frequency area from 50 to $1000 \mathrm{kHz}$ and at low power of electromagnetic field.

2. Studying the possibility of determining gas-hydrate formation beginning and measuring this process by means of a dielectric the parameters.

Description of the experimental mechanism. Experimental studies were carried out at the mechanism, which block diagram is shown in Figure 2. The main part of the mechanism is gas hydrate thermostatic chamber (reactor-crystallizer) with controlled gas supply system, as well as temperature and pressure control measuring instruments. Mechanism is designed for a pressure of about 1.6 MP and temperatures down to $-300 \mathrm{C}$. The main element of mechanism is a reactor (6) of high pressure where gas hydrates are either formed or dissociate under controlled conditions. Cylindrical condenser has a capacity about 88 sm3. Reagent is placed and gas is pumped into it. 
For the formation of hydrates in the reactor cell, it is necessary to maintain a certain range of temperatures and pressures. To modify and maintain the temperature thermostat KRIO-VT-01 is used (1). The pressure is maintained by means of the cylinder and valves $(2,12 \mathrm{a}, 12 \mathrm{~b})$. Pressure control is carried out with a sensor, which is attached to the reactor in a separate pipe. The temperature is monitored using a thermistor (5). After the experiment, the gas is vented from the system by opening the tap 12a, which also allows smoothen pressure release in the reactor for dissociation experiments.

To study the dielectric parameters of gas hydrates, electric circuit is hermetically mounted in the mechanism, consisting of a capacitor of cylindrical shape (7), mounting wires and kumetra (11) - measuring the quality factor - all this in general is an oscillation circuit.

The methodology of the experiment. In the experiments we used process water, as the hydrate-forming gas - propane. The stability of the hydrate formation process flow was supplied by maintaining temperature and pressure of certain conditions in reactor. In particular, in our experiments, the temperature was kept constant $(1.60 \mathrm{C})$ and a pressure was pumped cyclically to a constant maximum value exceeding the equilibrium pressure of the gas - gas hydrate - water system, at a given temperature. One cycle lasted 24 hours. Detailed methodology of increase kinetics and dissociation of gas hydrate is described in research [10]. In this work, a methodology measuring the dielectric parameters of gas hydrate in the process of growth and dissociation is presented for the first time.

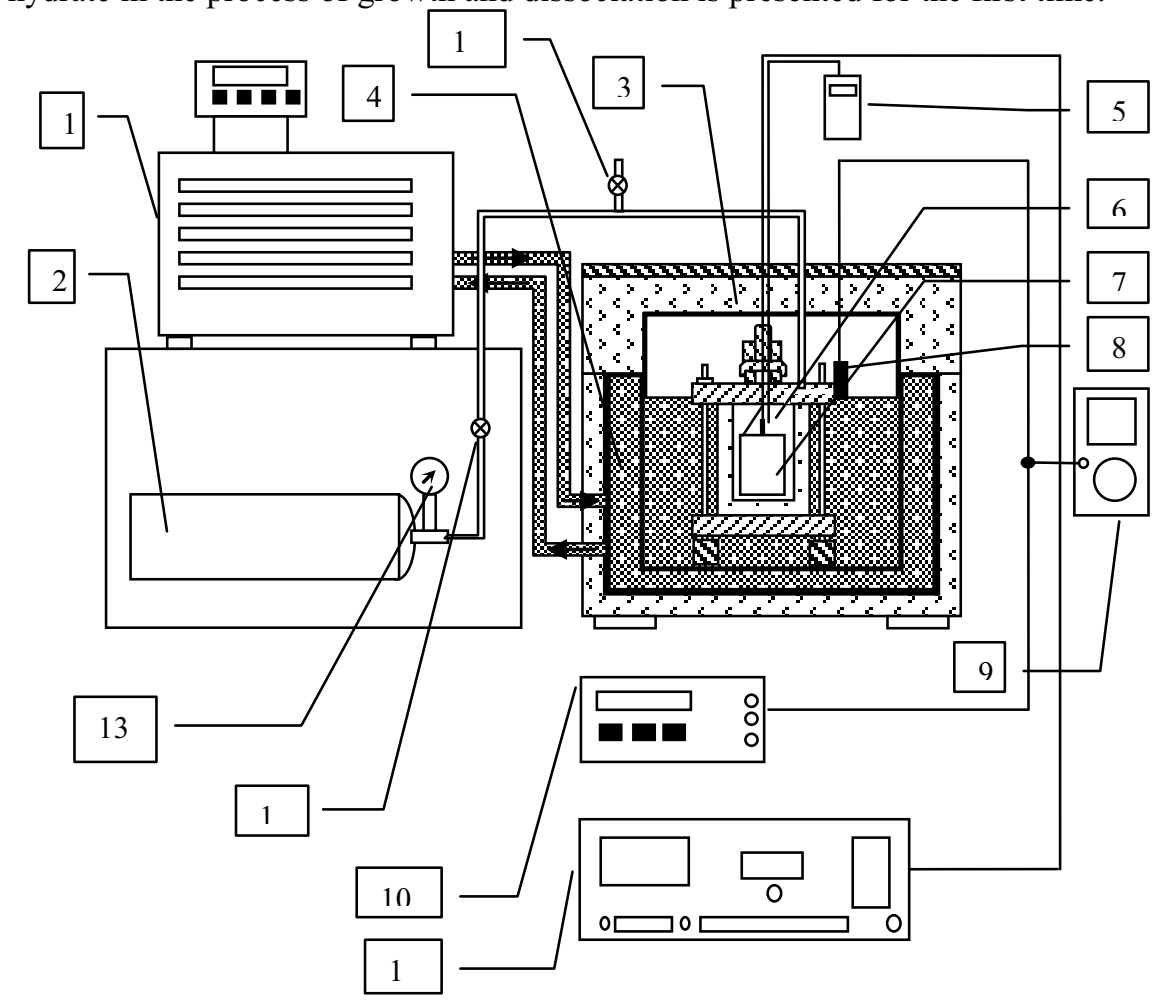

Fig. 2. The block diagram of mechanism

1 - thermostat KRIO-VT-01, 2 - a gas cylinder, 3 - cover of the heat exchanger, 4 - the first circuit of the heat exchanger, 5 - thermistor 6 - reactor 7 - cylindrical capacitor 8 - pressure sensor, 9 multimeter 10 - power source for the pressure sensor, 11 - Qmetr, 12a, 12b - acicular valves for pressure control, 13 -reduktor. 
The measurements of dielectric parameters were performed after each cycle using hydrate kumetra VM 560 in the range from $50 \mathrm{kHz}$ to $1 \mathrm{MHz}$. Measuring the dielectric loss tangent $\operatorname{tg}(\delta)$ and dielectric permittivity $\varepsilon^{\prime}$ were performed according to standard procedures [11]. In the jump of capacitance and quality factor of the oscillatory circuit, it can be judged on start of the formation of gas hydrate in the measuring cell. This determines the induction time of the beginning of the gas-hydrate formation and time of beginning measuring the dielectric parameters of gas hydrates. After the 1st cycle of hydrate formation, no signal was observed at kumetre, which is connected with the presence of significant amounts of liquid water and a small amount of gas hydrate in the measuring cell. After the second cycle of, the signal was reliably recorded. Since the temperature in the reactor is positive, and there is no ice in the measuring cell, the electromagnetic wave is mainly damping in gas hydrates. In subsequent cycles, with the accumulation of gas hydrate (dielectric) between the measuring capacitor plates, losses of electromagnetic energy are increasing. The greater the power dissipates in the dielectric, the larger the dielectric losses angle $\delta$ and the value of the function $\operatorname{tg}(\delta)$ are. Hence, can be judged not only on the presence of gas hydrate, but also its quantity at the value.

In the process of measuring the oscillation circuit parameters, the gas hydrate formed in the measuring cell heats. As experts' estimation shows, the rise in temperature must not exceed $0,8{ }^{\circ} \mathrm{C}[11]$ - in this case, the condition of the existence of gas hydrate complies. Therefore, the question arises about the temperature change in the measuring cell during the dielectric measurements. Our estimates of temperature change at kumetra power VM 560 and the length of the dielectric parameters measurements for 1.5 hours showed that the heat generated in the measuring cell (in the adiabatic approximation), is not enough to heat the gas hydrate even to decomposition temperature, not to mention the decomposition itself. Hence, gas hydrate decomposition does not occur in the dielectrical measurements.

Calculation of the mass of the formed gas hydrate in a cycle was determined experimentally from the ideal gas equation (PTV-method) according to the formula:

$$
\Delta m_{i}=\frac{\Delta P_{i} V \mu_{H}}{R T_{\gamma}}
$$

where $\Delta \mathrm{Pi}$ - pressure drop in a reactor during one cycle of hydrate formation, $\mathrm{Pa}, \mathrm{V}-$ the volume of the reactor, $\mathrm{R}$ - the gas constant; $\mathrm{T}$ - reactor temperature, $\mathrm{K}$; $\mathrm{i}$ - cycle number.

The mole fraction of hydrating agent in gas hydrates:

$$
\gamma=\frac{v_{G-H}}{v_{H}}
$$

where $v \mathrm{G}-\mathrm{H}$ - the number of moles of gas, which passed into gas hydrate; $v \mathrm{H}-$ the number of moles of gas hydrate. For propane, $\gamma=0.0555$ [14].

\section{Experiment Results and discussion}

Figure 3 shows photographs of gas hydrate grown in a cylindrical capacitor for 8 days (cycles). 

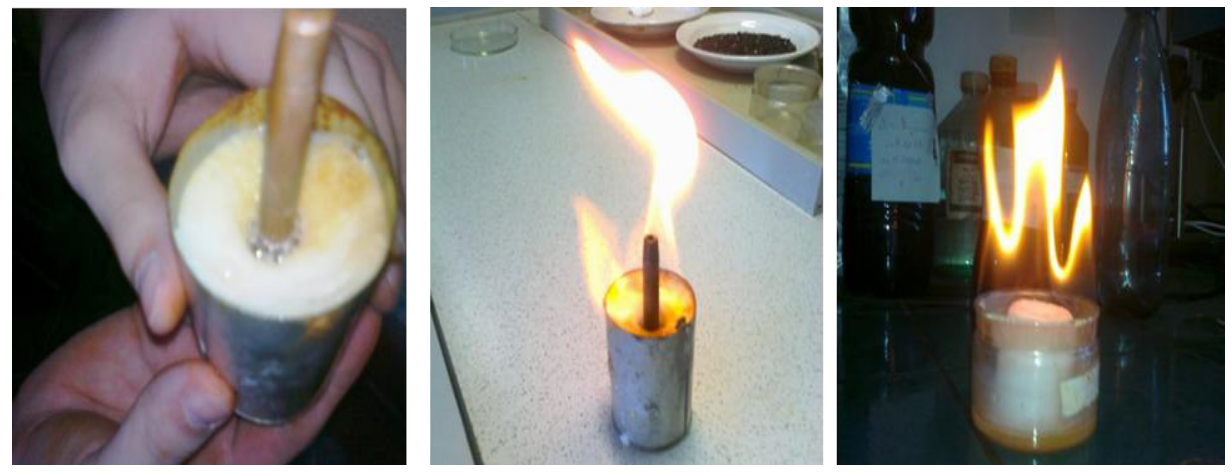

Fig. 3. Photos gas hydrate.

Figure 4 shows the dependence of the dielectric loss tangent of the frequency and number of a cycle at growth and dissociation of propane gas hydrate. The graph shows that with the growth of gas hydrate from cycle to cycle, the dielectric loss tangent increases, while dissociation - decreases. The frequency at which the kumetre signal is maximum, depends on the scheme, and the oscillating circuit parameters used in the experiment. In our case, the frequency is $\mathrm{f}=260 \mathrm{kHz}$.

Experimentally obtained values of dielectric loss tangent of gas hydrate allow to judge the degree of hydrate formation $\alpha$, which was determined experimentally, as the ratio of the dielectric loss tangent $i$-th cycle $\operatorname{tg}(\delta i)$ to the maximum tangent of the loss $\operatorname{tg}(\delta \max )$, where the water is completely converted to a gas hydrate:

$$
\alpha=\frac{\operatorname{tg}\left(\delta_{i}\right)}{\operatorname{tg}\left(\delta_{\max }\right)}
$$

In order to ensure consistency of the dielectric methods for quantifying the degree of gas-hydrate formation, it is necessary to compare it to another, independent from it, method. In this case, the simplest and most convenient method of comparison seems PVT method. Using the formula (1) we can calculate the degree of hydrate formation as:

$$
\alpha^{*}=\frac{\sum_{i=1} m_{i}}{m_{\max }}
$$

where mi - the gas hydrate mass, formed after i-th cycle, mmax - the total mass of the formed gas hydrate.

Figure 3 shows a comparison of dependencies of the degree on the time of hydrate formation obtained by two independent methods. It is clear from the graph, that the dielectric data are in good agreement with the results of the calculation by PVT method (difference does not exceed 10\%). 


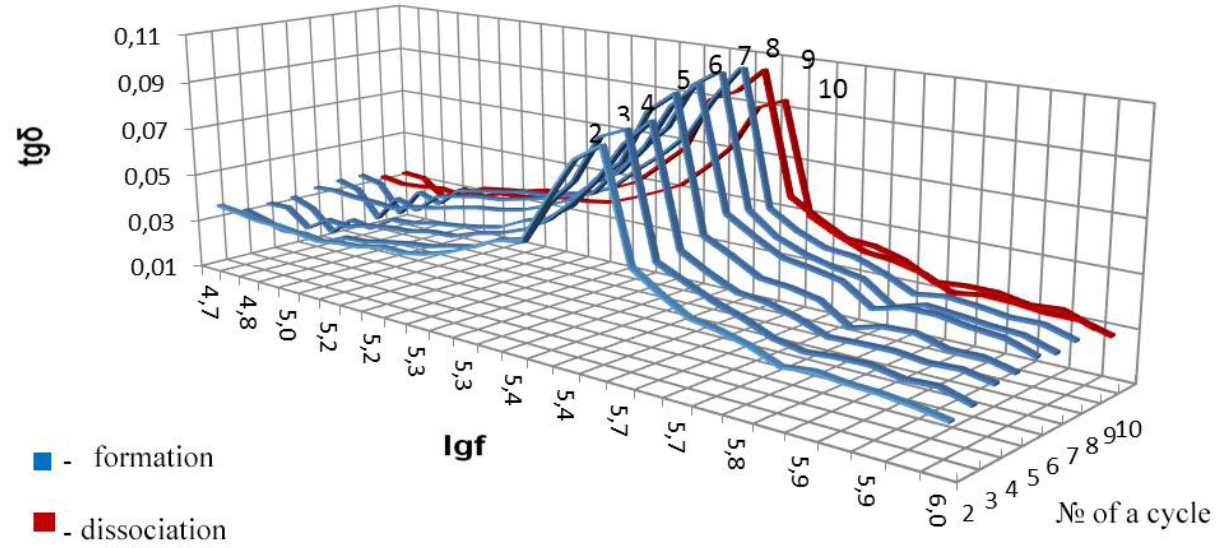

Fig. 4. The dependence of the dielectric loss tangent on the frequency and numbers of cycle in the formation and dissociation of gas hydrate.

In practical application [16-22] of surface-dielectric sensor, "dangerous" in terms of the start of hydrate formation, pipe section can be determined by the method developed in [15].

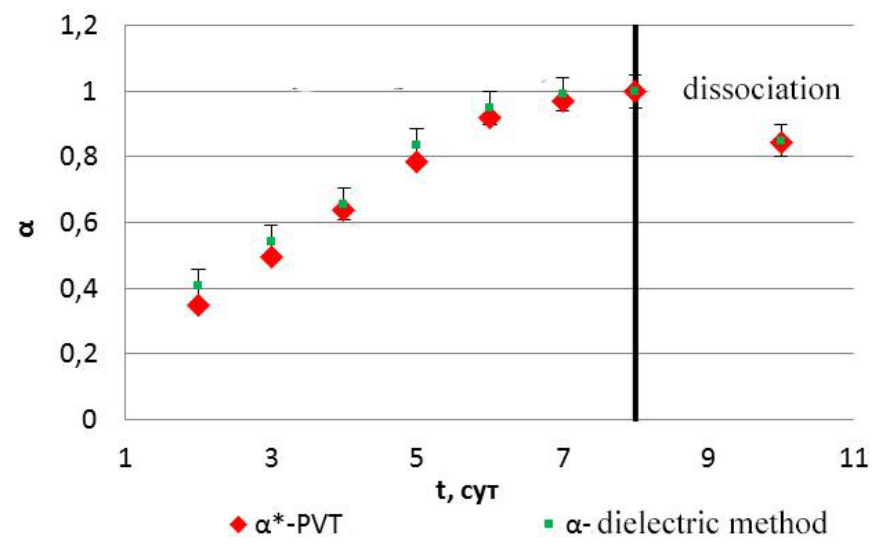

Fig. 5. The dependence of the degree hydrate formation on the time, obtained by two independent methods.

\section{Conclusion}

The results obtained allow offering a simple method of monitoring the gas hydrate formation process. This method is based on measuring the loss tangent at a certain frequency in the low frequency range of the electromagnetic field. The suggested method makes it possible to predict the time of safe operation of process equipment and avoid accidents.

\section{References}

1. Yu. Makogon, Geology and mineral resources of the oceans, 2, 9 (2010)

2. B. Degtyarev, B. Buchgalter, Fighting hydrates in the operation of gas wells in the northern regions (Nedra, Moscow, 1976) 
3. B. Beshevli, V. Savchenko, A. Kasyan, G. Kovalenko, Gas Industry, 2, 21-22 (1975)

4. T. Jakobsen, K. Folgerøzk. Meas. Sci. Technol, 8, 1006-1015 (1997)

5. M. Fatykhov, N. Bagautdinov, Oil and Gas Business, 15 (2006)

6. N. Bagautdinov, Dissertation of Doctor of Technical Sciences (2007)

7. S. Gough, Journ Phys Chem, 77(25), 2969 - 2976 (1973)

8. D. Davidson, Clatherate hydrates (NY Plenum Press, 1973)

9. D. Davidson, Can Journ Chem, 81, 248-253(1977)

10. A. Shabarov, A. Shirshova, S. Gasheva, Oil and gas, 2, 88-93 (2014)

11. Manual. Q-Meter VM 560. USSR, Brno TESLA Brno, Nat. entrepr. (1978)

12. D. Davidson, Clatherate hydrates (NY Plenum Press, 1973)

13. A. Groisman, Thermal properties of gas hydrates (Nauka, Novosibirsk, 1985)

14. D. Carroll, Natural gas hydrates (JSC "Premium Engineering", Moscow, 2007)

15. S. Buchinskiy, A. Shabarov, A. Burbasov, Vestnik TSU, 6, 28-33 (2008)

16. S. Stepanov, N., Cheremisin, S., Sokolov, A., Altunin, A., Shabarov, ECMOR 2012 13th European Conference on the Mathematics of Oil Recovery (2012) DOI: 10.399/2214-4609.20143270

17. V. Antip'ev, A. Nevolin, Yu. Zemenkov, Neftyanoe Khozyaistvo - Oil Industry, 10, 46-48 (1981)

18. V. Pavlov, K. Voronin, Yu. Zemenkov, Oil and Gas Terminal: Proceedings of the International scientific conference, 169-173 (2015)

19. S. Dudin, A. Shipovalov, S. Podorozhnikov, Yu. Zemenkov, Oil and gas, 2, 65-69 (2011)

20. Yu. Zemenkov, V. Shalay, M. Zemenkova, Procedia Engineering, 113, 254-258 (2015)

21. Yu. Zemenkov, V. Shalay, M. Zemenkova, Procedia Engineering, 113, 312-315 (2015)

22. R. Levitin, Yu. Zemenkov, Neftyanoe khozyaystvo - Oil Industry, 1, 110-114 (2016) 